\title{
On the Development of a Simple and Portable Ground-Based Infrared Imaging System for Lunar and Atmospheric Studies
}

\author{
Abdullrahman Maghrabi1 ${ }^{*}$, Georg Dittié2 , Abdulah Al-Dosari', Mohammed Al-Mutiri', \\ Mohammed Al-Tlasi' ${ }^{1}$, Abdulah Alshehri ${ }^{1}$ \\ ${ }^{1}$ The National Center for Applied Physics King Abdulaziz City for Science and Technology, Riydah, KSA \\ ${ }^{2}$ Dittié Thermografie, Königswinter, Germany \\ Email: *amaghrabi@kacst.edu.sa
}

How to cite this paper: Maghrabi, A., Dittié, G., Al-Dosari, A., Al-Mutiri, M., Al-Tlasi, M. and Alshehri, A. (2021) On the Development of a Simple and Portable Ground-Based Infrared Imaging System for Lunar and Atmospheric Studies. International Journal of Astronomy and Astrophysics, 11, 422-434.

https://doi.org/10.4236/ijaa.2021.113020

Received: August 4, 2021

Accepted: September 21, 2021

Published: September 24, 2021

Copyright $\odot 2021$ by author(s) and Scientific Research Publishing Inc. This work is licensed under the Creative Commons Attribution International License (CC BY 4.0).

http://creativecommons.org/licenses/by/4.0/ (c) (i) Open Access

\begin{abstract}
A small, portable, infrared (wavelength of $7-14 \mu \mathrm{m}$ ) system has been designed and developed to study the thermal behavior of the lunar surface and for thermal remote sensing applications. The principal operation of the system depends on collecting large amounts of infrared light, using a modified Newtonian telescope. The light from the object is reflected by the primary mirror and the secondary mirror. This collected light is then focused into a thermal camera by using an intermediate germanium lens as a field lens to provide a real optical image on the camera sensor. Several observations have been obtained out using the developed system, and eliciting some interesting results. These include lunar observations during different phases and during partial lunar eclipse. The thermal behavior of the lunar surface was identified, proving the system's functionality and performance. The developed system is, also, particularly suitable tool for outreach programs and students projects which can possibly offer useful learning and exploration opportunities for students in different applications. In this paper, a brief description about the developed system is provided. Some of the obtained results are illustrated. The future applications and improvements to the designed system are also summarized.
\end{abstract}

\section{Keywords}

Infrared, Optical-Thermal System, Moon, Atmosphere, Eclipse, Thermal Camera, Technology

\section{Introduction}

The moon being the closest celestial object to Earth has attracted scientists to 
study its geological structures and understand the physical and chemical properties of its surface, e.g., [1]-[6]. Detailed knowledge and data of lunar topography are of interest for planning lunar robotic and human explorations as well as establishing a potential platform for a range of scientific investigations [7] [8] [9] [10]. Moreover, lunar explorations are essential for fundamental and applied science, such as to evaluate the Earth, solar system and universe and conduct investigations in fundamental physics, astrobiology, and human physiology and medicine [11] [12] [13].

Optical observations have enabled astronomers to study the moon's surface at a scale far finer than is possible for any other object in the sky. Several topographical features have been identified on the moon's surface, including craters, domes, mountains, and dark halo and rubble areas, e.g. [14].

When radio telescopes and infrared (IR) detectors were developed in the mid- $20^{\text {th }}$ century, researchers were able to explore the lunar surface to a greater extent than was previously possible [15]-[23]. Additionally, direct investigation by space probes during the last half-century or so through space-based instruments [7] [21] [24] has also contributed to obtaining detailed information about the lunar surface.

Despite thermal, ground-based lunar observations having been conducted during the 1950s and 1960s using larger and professional telescopes, the utilization of this technology in recent times with respect to the technological developments in the field of thermal cameras and other associated accessories is limited. Considering this, we have succeeded in designing and developing a cost-effective and portable prototype for a ground-based infrared $(7-14 \mu \mathrm{m})$ system to study the thermal behavior of the lunar surface and use the flexibility this system which allows for the further applications of infrared remote sensing. The system was designed from theoretical considerations, without practical experience. However, the obtained results are acceptable and will allow us to further investigate and optimize the functionality and performance of the developed system. Being easy to use the developed system can be utilized for student projects and outreach programs and can be considered a useful tool for teaching thermography by qualitative visualization and/or quantitative analysis of a great number of physical phenomena [23] [25] [26] [27] [28].

In this paper, the basic design and the main components of the developed system are detailed in Section 1. Some of the obtained results are presented in Section 2. The conclusion and the future plans are discussed in the last section.

\section{The Detection System}

Figure 1 presents a schematic diagram of the IR-optical system. The principal operation of the system is to collect a large amount of light, using the primary mirror from the target object, and then reflect it onto the secondary mirror. This collected light is then focused into a thermal camera by using an intermediate germanium lens. Figure 2 shows the main components of the developed system, 
which will be briefly discussed in the following subsections.

\subsection{The Thermal Camera}

VarioCam IR, a microbolometer -based camera manufactured by Jenoptik, was used for the developed system. The camera contains a matrix of tiny resistors, which are warmed up by the incoming radiation. Even the slightest change in resistance can be scanned and measured by the camera. A big advantage of using this type of cameras is that the microbolometers technology does not need cooling and they are temperature stabilized. The camera has a focal plane array of $640 \times 420$ pixels and is sensitive to thermal radiations of $7-14 \mu \mathrm{m}$, with a temperature resolution of about $0.08 \mathrm{~K}$ at $30^{\circ} \mathrm{C}$. The camera delivers an image containing information about the IR intensity, which is stored on a SD card. The technical specifications of this camera are given in the datasheet of the camera (https://www.jenoptik.com/).

\subsection{The Newtonian Telescope}

A Newtonian telescope from Skywatcher Teleskop, of the type N 304/1500 PDS Explorer BD OTA, was adopted for this system. The telescope has a mechanical diameter of $360 \mathrm{~mm}$ and a length of more than $1500 \mathrm{~mm}$. This is necessary to concentrate the incoming IR light in to the aperture of the IR camera without optical losses or introducing image aberrations. It consists of a large concave

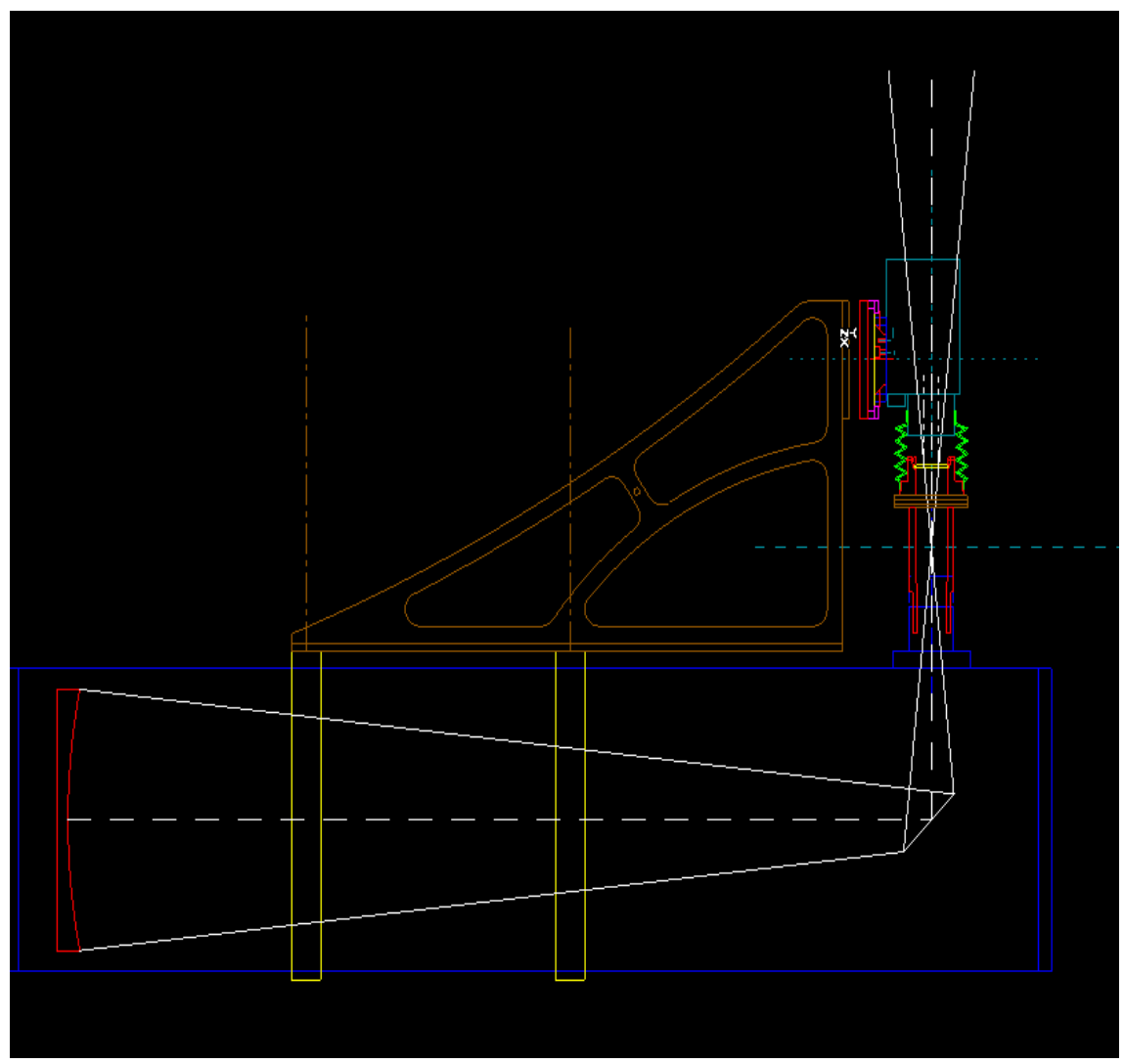

Figure 1. Schematic diagram showing the design of the proposed IR-optical system. 


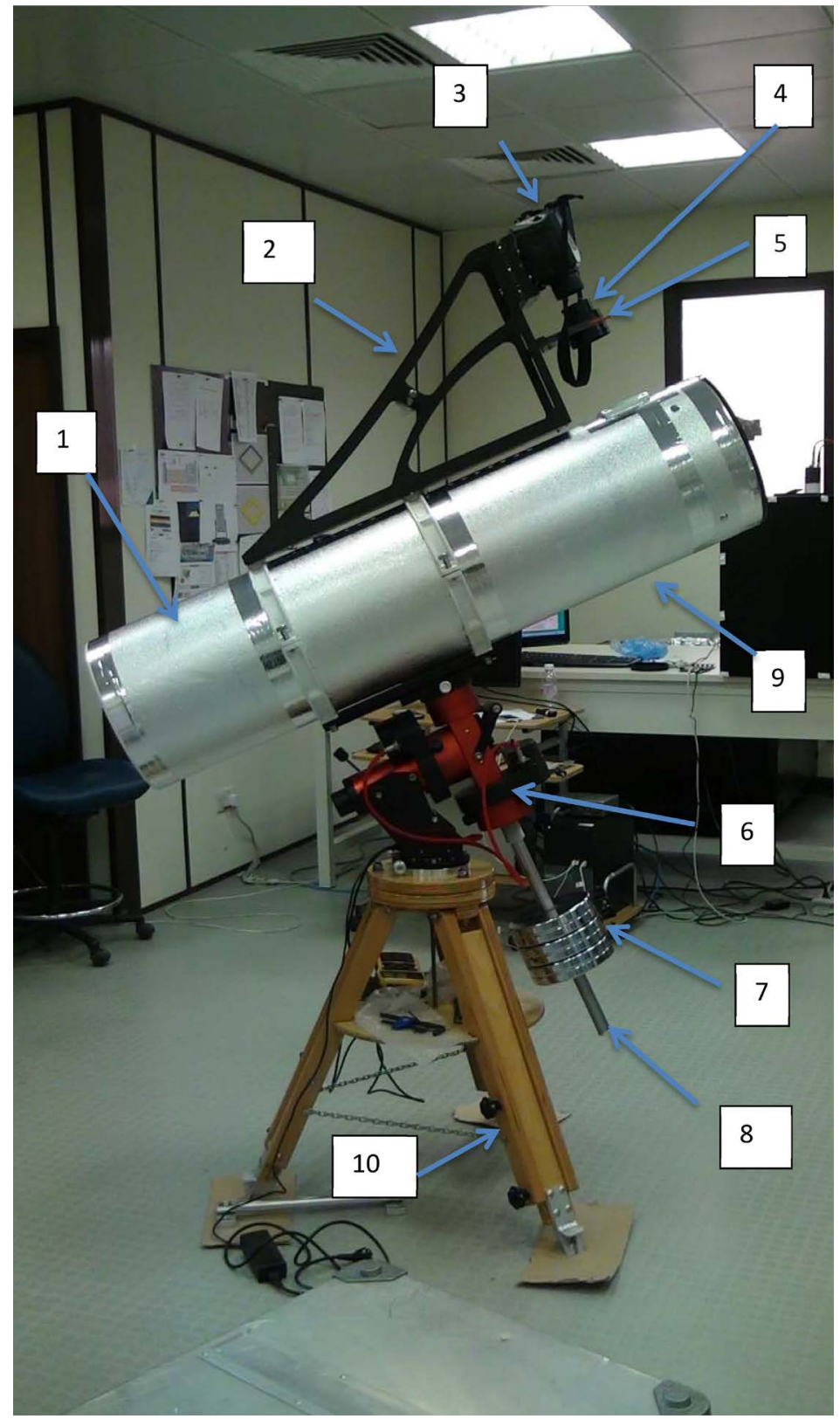

Figure 2. Shows the components of the developed system. 1 . Newtonian telescope; 2 . The Adjustable table; 3. The IR camera; 4. The Relay lens; 5 . The Safety shutter; 6 . The Equatorial mount; 7. The Counterweights; 8 . Weight rod holder; 9. Thermal blanket; 10. A sturdy tripod.

mirror and a flat secondary mirror. The main mirror forms an image at the relay lens, which projects it into the IR camera. The secondary mirror just bends the light out of the incoming light bundle to avoid large blocking by the camera. The overall field of view (FOV) of the optical system is $3 \times 2.3$ degrees, and the light gathering capacity is $1886 \mathrm{~mm}$ with a magnification of 608 .

\subsection{Germanium Relay Lens}

To form an image on a sensor, it is necessary to introduce a relay lens between 
the camera lens and the main mirror of the telescope. Only few materials are fully transparent in thermal infrared light. The most favorable material in this regard is germanium, which offers excellent chemical and mechanical stability as well as a hardness that allows machining with the demanded precision. The refractive index of germanium is very high, so an anti-reflection coating is crucial and can be found on all germanium surfaces. For the purpose of this project a Plano-convex a germanium lens with a diameter of $48 \mathrm{~mm}$ and an AR coating of 7 - 14 micron was used. This lens has a positive focal length and is best applied for infinite and finite conjugate applications (Figure 3).

\subsection{Mounting Rings and Adjustment Table}

The mounting rail carries two rings that hold the tube of the telescope. These rings are mounted with screws onto the rail, which also works as a stopper for the easy mounting of the whole assembly onto the equatorial mount (Figure $5(\mathrm{a}))$.

We have mechanically designed an adjustment table to carry the IR camera and mount it in its desired position. This table has a dedicated plate to mount the camera from which an exact view of the relay lens is available. A dedicated adaptor was designed to hold the camera on the plate, and it allows us to mount the camera on the vertical side of the adjustment table as it looks down into the relay lens. The adjustment ring offers all the necessary freedom grades like shift and tilt angles to put the IR camera into its proper position. Once adjusted, the position of the camera has to be checked, but no realignment is needed in general.

A red colored safety shutter was introduced in front of the relay lens to prevent the destruction of the camera by sunlight when the sun used as a calibration object. It is fixed by magnets in open and closed positions, so no extra fixing is needed, and it can be moved at the beginning with observation.

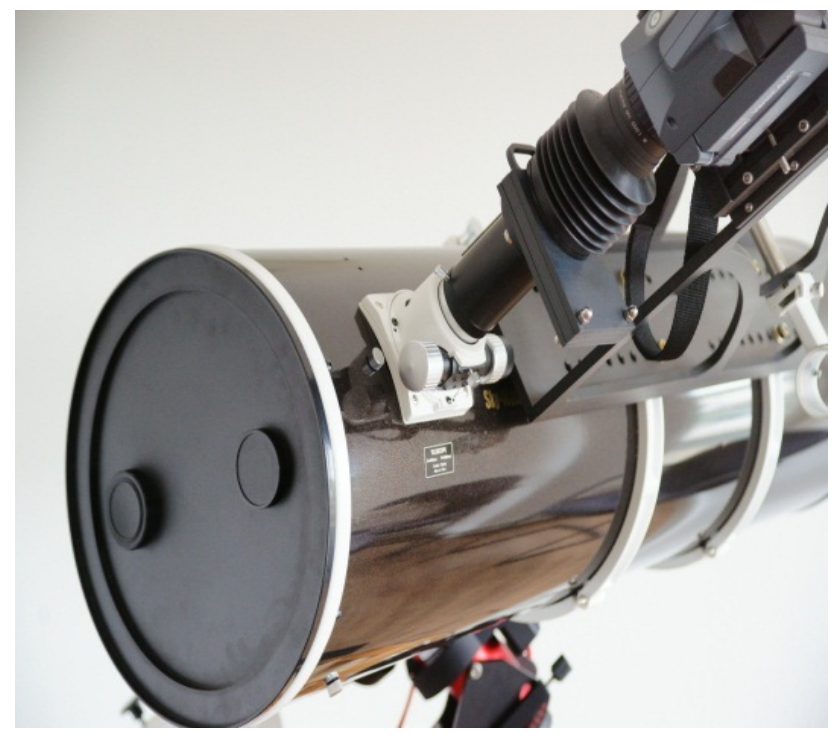

Figure 3. The Germanium lens attached to its base, located between the IR camera and the optical telescope. 


\subsection{The Equatorial Mount and the Tripod}

The assembly of the IR camera, adjustment table, Newtonian telescope and the relay lens group with safety shutters are big and heavy. For pointing to a given position, a matching mount with sufficient carrying capacity is needed. Commercial fork mounts of altazimuth style are not available for long, big telescopes like this. For the purpose of this project, the Rainbow Astro Morning Calm 200 GE equatorial mount with high tracking accuracy ( \pm 5 arcsecond) was used. The mount has a dedicated controller that contains a microcontroller for doing position calculations, driving the two positioning drives, and tracking the daily movement of the celestial objects.

To ensure the function of automatic positioning, a sturdy tripod was designed to carry the weight of the mount together with the counterweights, telescope and the camera including the adjustment table. The tripod has three legs that can be individually extended to put the mounting top of the tripod into an exact horizontal position.

\subsection{Image Processing}

For the purpose of this project, a dedicated version of the "Fornax" software was development and utilized for image processing. This commercial software was initially developed by Dr. George Dittié for thermography and image processing. The dedicated version of this software incorporated several image processing routines to get a better image quality for the presentation. For instance, the software can reduce the noise of the thermal observations by averaging a row of raw thermograms (thermal images). The signal to noise ratio is increased by the square root of the average number of thermograms. Because the target object can move somewhat in the field of view the image can be re-centered before being superimposed to the average image, hence, the image blur due to slight misalignments or mechanical errors can be rejected. Moreover, the vignetting effect can be removed using the field flattening function available in the software. This function subtracts a background with superimposed vignetting limb from the thermal image. Besides, the software also allows the user to select specific part of the processed image and plot temperature profiles.

\section{Results}

The developed system has been tested, calibrated, and used in several practical experiments to ensure its functionality and performances using different targets.

We began the testing state by implementing signature imaging for some well-known targets such as buildings and towers. These experiments allow us to understand the thermal behavior of the studied objects. Moreover, these studies will enable us to understand the atmospheric absorption/emission and its influence on the observations during different atmospheric conditions [29].

Figure 4 is an example shows the thermal and optical images of Alfayslia tower. This tower is part of the Riyadh skyline and is about $7 \mathrm{~km}$ from the 
observation site. The thermal image clearly shows the structure of the building, where the red spots indicate the warmer parts of the tower.

Figure 5 shows the thermal images of the moon during different lunar phases. It obvious that the temperature distributions on the surface of the moon are

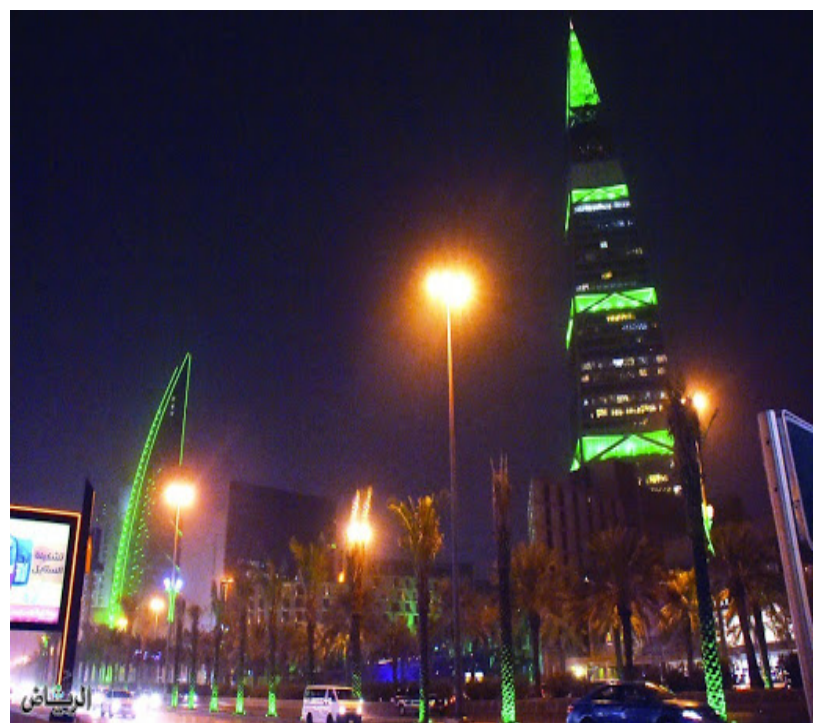

(a)

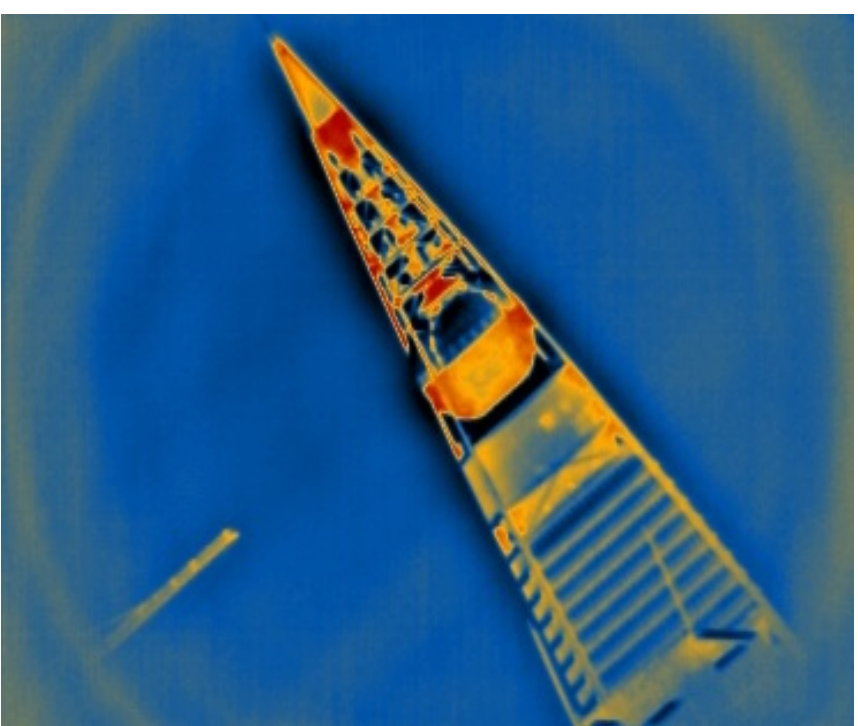

(b)

Figure 4. Shows (a) the Optical and (b) the Thermal images of the Alfayslia tower.

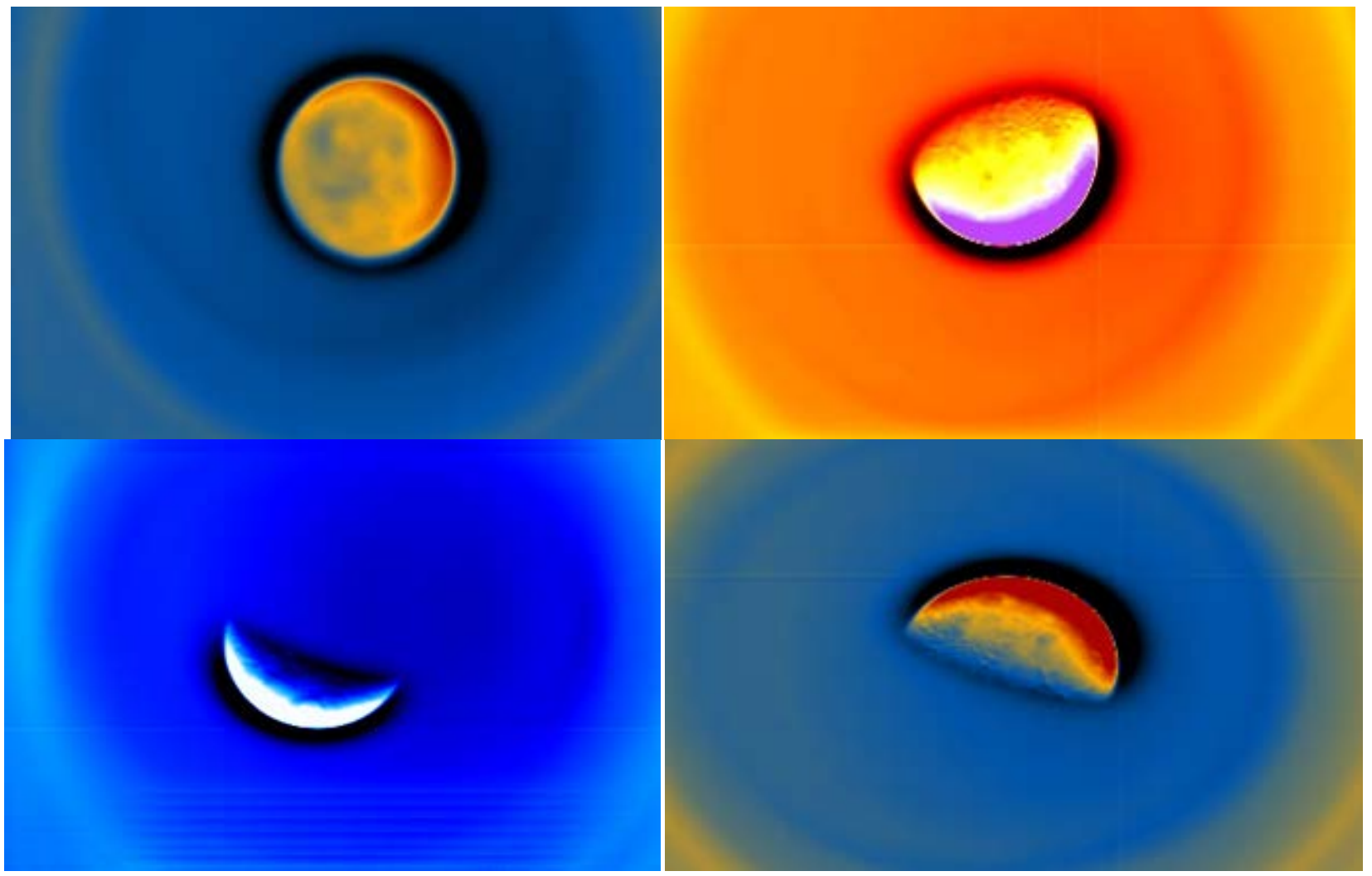

Figure 5. Shows the thermal images of the moon during different phases: Top left (30 August 2016), Top right (11 March 2015), Bottom left (3 May 2016), and Bottom right (8 July 2015). 
affected by the illuminated part of the lunar surface. Some of the structures of the lunar surface, such as craters and mountains are clearly seen and their temperatures were different from one phase to another [24] [27] [28] [30] [31].

Figure 6(a), shows the thermal image of the crescent observed on the $21^{\text {st }}$ of January 2015 at 4:37 PM whereas Figure 6(b), captures the illumination of the moon at the time of observation. The astronomical ephemeris of the moon at the time of observation showed that the moon was a 26 -h old moon, with an altitude of $17^{\circ} 47^{\prime}$ and an angular separation of $19^{\circ} 54^{\prime}$, phase $3.6 \%$ at air mass of 3.27 , which shows the capabilities of the developed system in detecting the thin crescent. The obtained results of the crescent observation can be of interest to those who are looking for alternative techniques of observing the crescent. However, the obtained results are preliminary and more practical sessions are suggested to attain the world record of crescent citing.

Figure 7(a), shows the thermal image of the planet Mercury obtained on January 22, 2015. The illustration of the position of Mercury at the time of observation was obtained from the Sky software and is presented in Figure 7(b). The observation time was 5:10 PM, two minutes after the sunset, the altitude was 13 19 , phase $21.1 \%$, air mass 4.30 , and the angular separation from the sun was 17 34. The success of these initial practical experiments demonstrated the principal functionality of the IR telescope.

Additionally, using the developed system, we were able to observe the partial lunar eclipse that occurred on September 27, 2015. Figure 8, shows some of the obtained thermal images during this eclipse.

It is obvious from the images that the highlands (e.g., Moon Alps) and younger craters like Copernicus and Tycho on the moon are brighter than, for instance, darker lava plains. This is partly because the former features absorb less

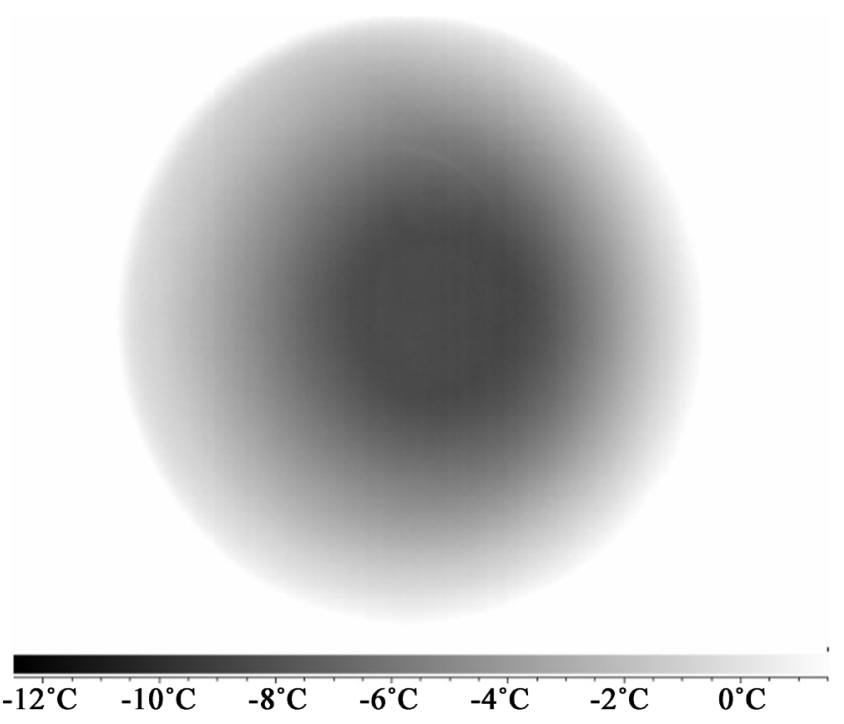

(a)

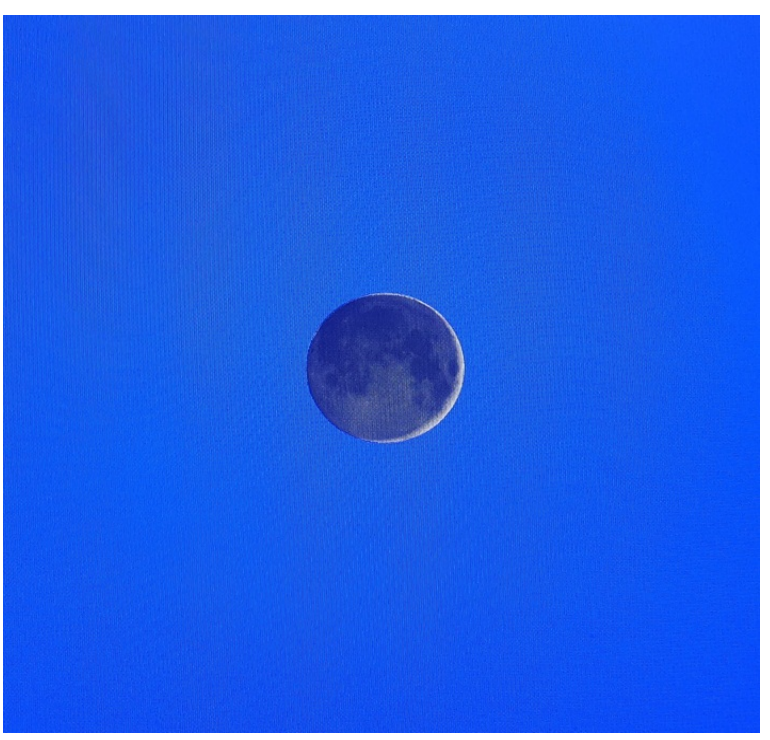

(b)

Figure 6. (a) Shows the thermal image of the 26 -h old crescent recorded on the $22^{\text {nd }}$ January 2015 and (b) is presentation of the illuminated part of the moon at the time of the observation. 
solar radiation than the latter. At thermal infrared wavelengths, the lunar surface is nearly an ideal black-body radiator, mostly due to the tiny spaces and holes between the dust grains [6] [24] [28] [32] [33] [34]. The thermal properties of the dust layer on the lunar surface are responsible for the large temperature changes during a lunar day [31]. Therefore, lunar eclipses present excellent opportunities to study the changes that happen on the lunar surface when solar

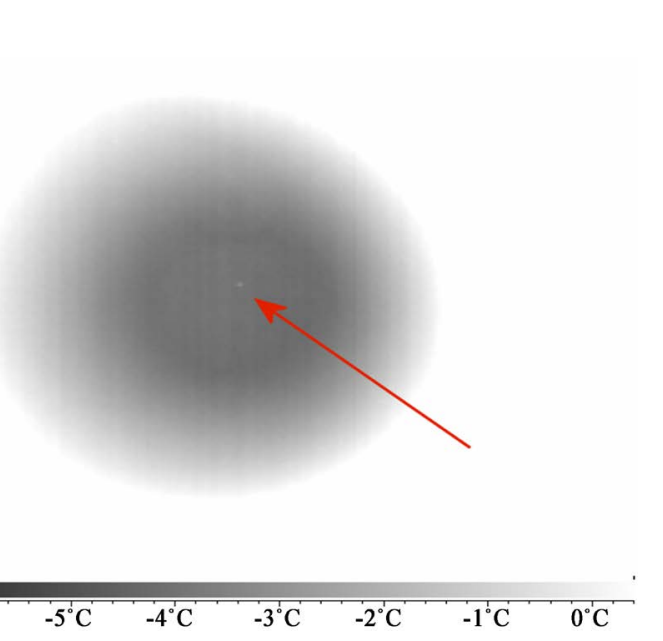

(a)

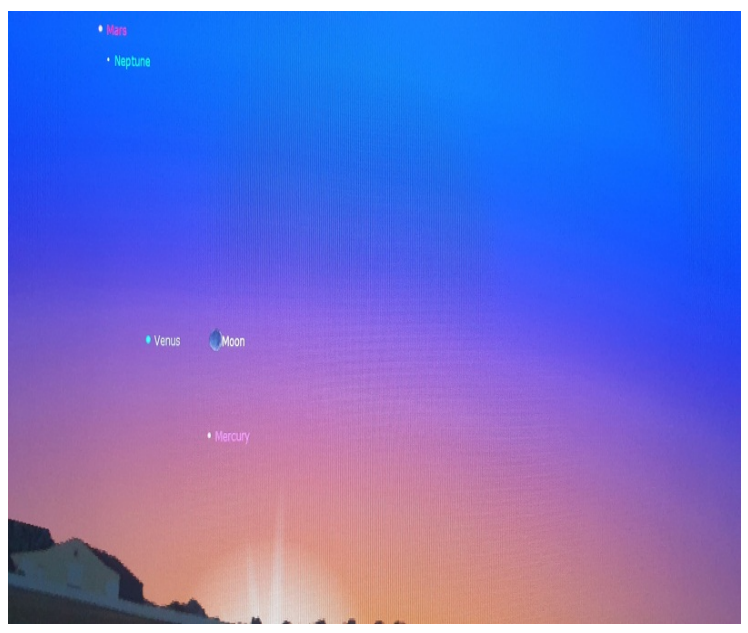

(b)

Figure 7. (a) Shows the first thermal images of Mercury obtained from observation session conducted in $22^{\text {nd }}$ January 2015 , Riyadh. (b) Sky software illustration of the positions of Mercury and other planets at the time of observation.

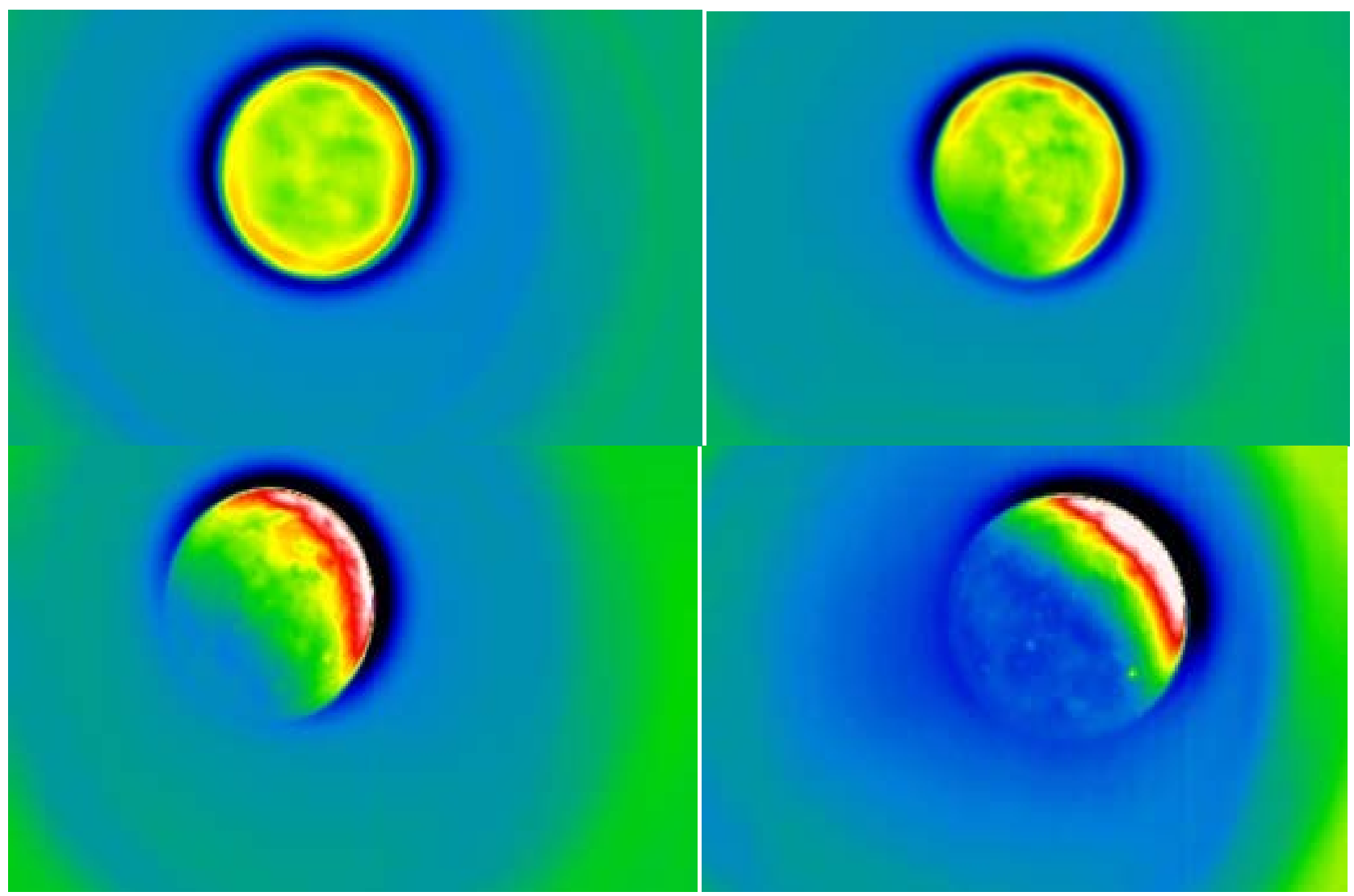




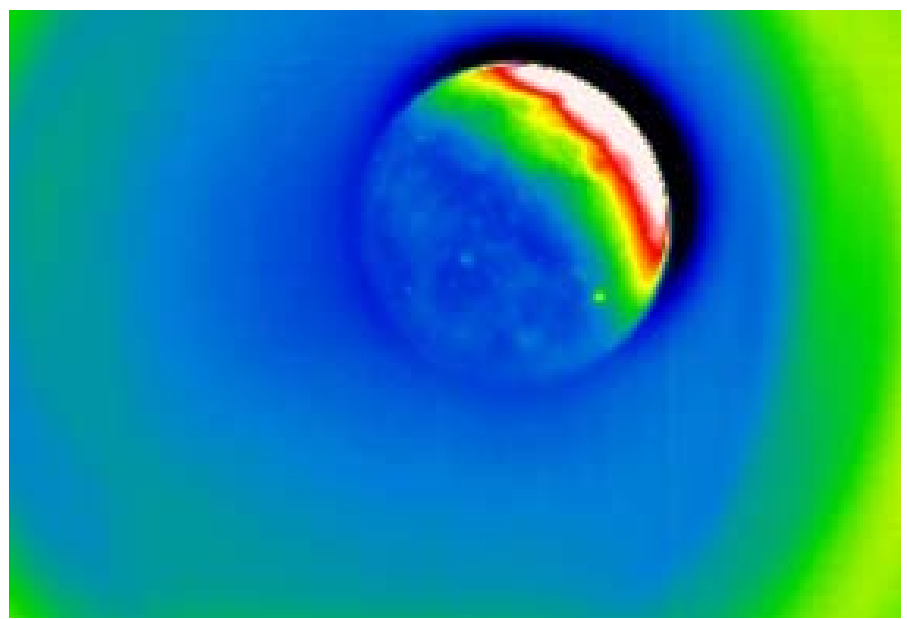

Figure 8. Shows the different thermal images taken by the developed system in Riyadh during the lunar eclipse that occurred on the $27^{\text {th }}$ September 2015. In this eclipse, we were able to observe the first part of the eclipse from full to totally eclipsed moon. However, the moon set when it was totally eclipsed; hence we were not able to observe the second phase of the eclipse.

radiations are suddenly cut off by the transit of the Earth [31] [35]. Thermal properties of the lunar surface during different lunar eclipses obtained by the developed system constitute the subject of ongoing project.

\section{Conclusions and Future Plans}

A prototype portable infrared $(7-14 \mu \mathrm{m})$ system has been designed, developed, and used for lunar and atmospheric studies. The system is one of the ground-based thermal imaging instrumentations which utilize the advance technology of thermal cameras and associated accessories.

Preliminarily tests have been conducted under real conditions and have shown good results. Thermal observations for the lunar surface were carried out in different phases and showed superior results. Lunar features like the highlands and particularly, the less dark cratered lowlands were clearly identifiable. Up till now, the telescopic system was probably sensitive enough to detect the stored warmth from the past lunation on the bottom of younger craters. Additionally, a 26-h old crescent has been clearly cited without any complications and the thermal image of Mercury is obtained.

The scientific importance of the IR telescope lies not only in finding out more about the thermal behavior of the lunar surface but also uses the flexibility of the system to study more about further applications of IR systems in remote-sensing applications. The system can be used for non-destructive testing from a distance, such as on power lines or very large constructions like bridges and towers. A notable application of the system is that it can be used to learn more about our atmosphere, particularly about aerosols, by exploring the atmospheric absorption and emission in thermal IR wavelengths. Measuring the atmospheric absorption and emission by comparing the signal with the given lunar temperature at different altitudes above the horizon can deliver valuable information about our atmosphere. This can be very important for improving climate simulations, 
particularly for the subtropical zones. Additionally, the developed system can be used for student's projects and outreach programs which can offer rich learning and exploration opportunities for students and researchers in different fields. These include detector technologies and optics, involving in some meaningful measurements to understand the thermal properties of the moon and the atmosphere. In such programs students and researchers will involve in the world of experimental thermal imaging, calibration procedures, data analysis and interpretation, and, finally, the sharing and distribution of the scientific results.

Since the actual version of the telescope is a prototype designed without practical experience, based on theoretical considerations. It works well, but there is still room for further enhancements that build on the experiences gained by the first operation of the prototype. There are several modifications which have been suggested for the better performance and functionality for the developed system. These relate to the limitations of the size of the relay lens, diameter of the optical mirror, alignments, and weight of the whole system.

For extended use, the construction of a sturdy permanent pier instead of a tripod would increase the mechanical precision and stiffness by an order of 10 . The actual telescope is sensitive within the given limits of the IR camera and the atmospheric range, but this is true only for the circle of the innermost field of view, which covers $2 / 3^{\text {rd }}$ of the vertical dimensions of the camera sensor. The majority of the field is affected by vignetting. This is caused by the limited clear diameter of the germanium relay lens. Despite the vignetting, the developed system can be used without problems but only within a limited field of view. The limited diameter is a consequence of the budget limits imposed for the prototype to reduce the economic risk.

More frequent observational sessions are suggested to overcome the environmental problems. These observations will help in establishing a reliable database of atmospheric behavior under different circumstances, which will be useful for the scientific community.

\section{Acknowledgements}

We would like to thank the King Abdulaziz City for Science and Technology (KACST) for supporting this work. Also, we would like to thank the anonymous reviewers for their valuable comments and recommendations.

\section{Conflicts of Interest}

The authors declare no conflicts of interest regarding the publication of this paper.

\section{References}

[1] Burns, J.O., Duric, N., Taylor, G.J. and Johnson, S.W. (1990) Observatories on the Moon. Scientific American, 262, 18-25. https://doi.org/10.1038/scientificamerican0390-42

[2] Vogler, K., Johnson, P. and Shorthill, R. (1991) Modeling the Non-Grey-Body Thermal Emission from the Full Moon. Icarus, 92, 80-93.

https://doi.org/10.1016/0019-1035(91)90037-T 
[3] Wieczorek, et al. (2006) The Constitution and Structure of the Lunar Interior. Reviews in Mineralogy and Geochemistry, 60, 221-364. https://doi.org/10.2138/rmg.2006.60.3

[4] Jaumann, R., et al. (2012) Geology, Geochemistry and Geophysics of the Moon: Status and Current Understanding. Planetary and Space Science, 74, 15-41. https://doi.org/10.1016/j.pss.2012.08.019

[5] Vasavada, A.R., Bandfield, J.L., Greenhagen, B.T., et al. (2012) Lunar Equatorial Surface Temperatures and Regolith Properties from the Diviner Lunar Radiometer Experiment. Journal of Geophysical Research, 117, E00H18. https://doi.org/10.1029/2011JE003987

[6] Williams, J., Paige, D., Hayne, P. and Siegler, M. (2012) The Influence of Surface Roughness and Rocks in LRO Diviner Observations. NASA Lunar Science Forum, NASA Ames.

[7] Vaniman, D., Dietrich, J., Taylor, G. and Heiken, G. (1991) Exploration, Samples and Recent Concepts of the Moon. In: Heiken, G., Vaniman, D. and French, B., Eds., Lunar Sourcebook. A User's Guide to the Moon, Cambridge University Press, Cambridge, 5-26.

[8] McEwen, A. and Robinson, M. (1997) Mapping of the Moon by Clementine. Advances in Space Research, 19, 1523-1533. https://doi.org/10.1016/S0273-1177(97)00365-7

[9] Crawford, I.A. and Zarnecki, J. (2008) Astronomy from the Moon. Astronomy \& Geophysics, 49, 2.17-2.19. https://doi.org/10.1111/j.1468-4004.2008.49217.x

[10] Vondrak, R., Keller, J., Chin, G. and Garvin, J. (2010) Lunar Reconnaissance Orbiter (LRO): Observations for Lunar Exploration and Science. Space Science Reviews, 150, 7-22. https://doi.org/10.1007/978-1-4419-6391-8 3

[11] Nozette, S., et al. (1994) The Clementine Mission to the Moon: Scientific Overview. Science, 266, 1835-1839. https://doi.org/10.1126/science.266.5192.1839

[12] Cockell, C.S. (2010) Astrobiology: What Can We Do on the Moon? Earth Moon Planets, 107, 3-10. https://doi.org/10.1007/s11038-010-9363-2

[13] Crawford Ian, A. and Joy Katherine, H. (2014) Lunar Exploration: Opening a Window into the History and Evolution of the Inner Solar System. Philosophical Transactions of the Royal Society A, 372, Article ID: 20130315.

[14] Shkuratov, Y., Kaydash, V., Korokhin, V., Velikodsky, Y., Opanasenko, N. and Videen, G. (2011) Optical Measurements of the Moon as a Tool to Study Its Surface. Planetary and Space Science, 59, 1326-1371. https://doi.org/10.1016/j.pss.2011.06.011

[15] Pettit, E. and Nicholson, S. (1930) Lunar Radiation and Temperatures. The Astrophysical Journal, 71, 102-135. https://doi.org/10.1086/143236

[16] Piddington, J. and Minnett, H. (1949) Microwave Thermal Radiation from the Moon. Australian Journal of Scientific Research. Series A, 2, 63. https://doi.org/10.1071/CH9490063

[17] Ryadov, V.Y., Furashov, N.I. and Shronov, G.A. (1964) Measurements of the Moon's Natural Infrared Thermal Radiation. Soviet Astronomy Journal, 8, 82-85.

[18] Drake, F. (1965) The Nature of the Lunar Surface. In: Hess, W.N., Menzel, D.H. and O'Keefe, J.A., Eds., Radio Measurements of the Moon, Johns Hopkins Press, Baltimore, Chap. 17, 277.

[19] Saari, J. and Shorthill, R. (1966) Review of Lunar Infrared Observations. Boeing Scientific Research Laboratories, Document D1-B2-05B6. 
[20] Ulich, B.L., Cogdell, J.R., Davis, J.H. and Calvert, T.A. (1974) Observations and Analysis of Lunar Radio Emission at 3.09 mm Wavelength. The Moon, 10, 163-174. https://doi.org/10.1007/BF00655717

[21] Paige, D.A., Foote, M.C., Greenhagen, B.T., Schofield, J.T., et al. (2009) The Lunar Reconnaissance Orbiter Diviner Lunar Radiometer Experiment. Space Science Reviews, 150, 125-160. https://doi.org/10.1007/978-1-4419-6391-8 7

[22] Vollmer, M. and Mollmann, K. (2012) Surface Temperatures of the Moon Measurements with Commercial Infrared Cameras. European Journal of Physics, 33, 1703-1719. https://doi.org/10.1088/0143-0807/33/6/1703

[23] Shaw, J., Nugent, P. and Vollmer, M. (2015) Infrared Moon Imaging for Remote Sensing of Atmospheric Smoke Layers. Applied Optics, 54, B64-B75. https://doi.org/10.1364/AO.54.000B64

[24] Lawson, S.L., Jackosky, B.M., Park, H.-S. and Mellon, M.T. (2003) Brightness Temperatures of the Lunar Surface: Calibration and Global Analysis of the Clementine Long-Wave Infrared Camera Data. Journal of Geophysical Research, 105, 4273-4290. https://doi.org/10.1029/1999JE001047

[25] Möllmann, K.P. and Vollmer, M. (2007) Infrared Thermal Imaging as a Tool in University Physics Education. European Journal of Physics, 28, S37-S50. https://doi.org/10.1088/0143-0807/28/3/S04

[26] Möllmann, K.-P. and Vollmer, M. (2010) Infrared Thermal Imaging: Fundamentals, Research and Applications. Wiley, Hoboken.

[27] Möllmann, K.-P. and Vollmer, M. (2013) Characterization of IR Cameras in Student Labs. European Journal of Physics, 34, S73-S90. https://doi.org/10.1088/0143-0807/34/6/S73

[28] Rashman, M., Steele, I., Bates, S., Copley, D. and Longmore, S. (2020) Uncooled Microbolometer Arrays for Ground-Based Astronomy. MNRAS, 492, 480-487. https://doi.org/10.1093/mnras/stz3497

[29] Maghrabi, A.H. (2012) Modification of the IR Sky Temperature under Different Atmospheric Conditions in an Arid Region in Central Saudi Arabia: Experimental and Theoretical Justification. Journal of Geophysical Research, 117, D19207. https://doi.org/10.1029/2012JD017881

[30] Feng, J.Q., et al. (2010) Review on Physical Models of Lunar Brightness Temperature. Chinese Journal of Geochemistry, 29, 204-211. https://doi.org/10.1007/s11631-010-0204-9

[31] Maghrabi, A. (2014) On the Measurements of the Moon's İnfrared Temperature and İts Relation to the Phase Angle. Advances in Space Research, 53, 339-347. https://doi.org/10.1016/j.asr.2013.10.022

[32] Shorthill, R.W. and Saari, J.M. (1965) Radiometric and Photometric Mapping of the Moon through a Lunation. Annals of the New York Academy of Sciences, 123, 772-739. https://doi.org/10.1111/j.1749-6632.1965.tb20396.x

[33] Linsky, J. (1966) Models of the Lunar Surface Including Temperature-Dependent Thermal Properties. Icarus, 5, 606-634. https://doi.org/10.1016/0019-1035(66)90075-3

[34] Sonett, C.P. (1982) Electromagnetic Induction in the Moon. Reviews of Geophysics and Space Physics, 20, 411-455. https://doi.org/10.1029/RG020i003p00411

[35] Saari, J.M., Shorthill, R. and Deaton, T. (1966) Infrared and Visible Images of the Eclipsed Moon of December 19, 1964. Icarus, 5, 635-659. https://doi.org/10.1016/0019-1035(66)90076-5 